\title{
Gastrocopta iheringi (Suter, 1900) (Gastropoda, Vertiginidae): redescrição do material-tipo
}

\author{
Inga L. Veitenheimer-Mendes* \\ Carla Regina Pedroso de Oliveira
PPG em Avaliação de Impactos Ambientais em Mineração e de Memória Social e Bens Culturais UNILASALLE, Avenida Victor Barreto, 2288, CEP 92010-000. Canoas - RS, Brasil
*Autor para correspondência
inga.mendes@terra.com.br

Submetido em 18/07/2011

Aceito para publicação em 22/10/2011

\section{Resumo}

Redescreve-se o material-tipo de Gastrocopta iheringi (Suter, 1900), sendo confirmado e ilustrado o holótipo. São elucidadas questões relativas à identificação de micromoluscos do gênero Gastrocopta Wollaston, 1878.

Palavras-chave: Brasil, Holótipo, Localidade-tipo, Pupa iheringi, Rio Grande do Sul

\section{Abstract}

Gastrocopta iheringi (Suter, 1900) (Gastropoda, Vertiginidae): redescription of type material. The holotype of Gastrocopta iheringi (Suter, 1900) was redescribed, illustrated and its identity was confirmed. The goal of this work was to elucidate questions relating to the identification of micromollusks in the genus Gastrocopta Wollaston, 1878.

Key words: Brazil, Rio Grande do Sul States, Pupa iheringi, Holotype, Type-locality

\section{Introdução}

Malacólogo residente na Nova Zelândia, Suter recebe de Herman von Ihering conchas provenientes de ambientes terrestres do Brasil, e em 1900 descreve para a família Pupidae [sic] o táxon Pupa iheringi, informando: "Typo na minha collecção. - Hab. Cidade de Rio Grande do Sul (Bollaxa), tirado em estado subfossil de deposito moderno." Ilustrou o novo táxon a partir de desenhos de: "concha em vista frontal (Fig. 8) e detalhe da abertura (Fig. 8a)".
Pilsbry (1916-1918) ao revisar a família Pupillidae transfere Pupa iheringi para o gênero Gastrocopta Wollaston, 1878, fornecendo caracterização e ilustração de suposto parátipo, segundo ele, depositado na Academy Natural Sciences of Philadelphia (ANSP). Pilsbry (19161918) relaciona, ainda, 12 espécies de Gastrocopta para a América do Sul, das quais apenas G. iheringi é citada para o sul do Brasil. Morretes (1949), em seu catálogo dos moluscos do Brasil, relaciona G. iheringi e informa a mesma distribuição da descrição original: "Bolaxa, Rio Grande do Sul (v. Ihering)". 
Marshall (1996), em seu artigo sobre tipos depositados no Museum of New Zealand Te Papa Tongarewa (NMZN), relaciona um lote como: "Pupa iheringi Suter, 1900:336. Syntypes (2) M.205848. Gastrocopta (Immersidens)."

Thompson e Lopéz (1996) colocam em dúvida o registro de $G$. iheringi para locais diferentes do fornecido por Suter (1900), isto é, extremo sul do Brasil. Os autores mencionam os registros feitos em 1940 por Richards e Hummelinck para a Venezuela e ilhas do Caribe localizadas na costa desse país, incluindo a Ilha Margarita. Entretanto, observam que é preciso um exame cuidadoso dos espécimes para confirmar tais registros. Thompson e Lopéz (1996) descrevem Gastrocopta (Immersidens) gularis, como uma nova espécie para a Nicarágua e mencionam que características da concha seriam semelhantes às das espécies sul-americanas $G$. colombiana Pilsbry, 1921, G. humellink Hass, 1960 e G. iheringi.

Miguel e Parent (1996), em levantamento da malacofauna de água doce e terrestre da província de Santa Fé, Argentina, registram G. iheringi como nova citação para Santa Fé, no entanto, informam que a identificação não é precisa, devido à falta de tipos, ilustrações e adequadas descrições destes micromoluscos.

Salgado e Coelho (2003), em seu catálogo dos moluscos terrestres brasileiros, relacionam $G$. iheringi citando como trabalhos consultados Suter (1900) e Morretes (1949).

Simone (2006), em seu catálogo dos moluscos terrestres e límnicos do Brasil, com base em lotes depositados no Museu de Zoologia da Universidade de São Paulo (MZSP 32872 e MZSP 7519), que considera como prováveis síntipos, disponibiliza as fotos dos micromoluscos e os registra como Gastrocopta iheringi (Suter, 1900) n.comb.

A redescrição e as fotomicrografias dos tipos de G. iheringi, objetivam auxiliar a elucidar questões relativas à identificação de micromoluscos do gênero Gastrocopta, especialmente aqueles que têm sido identificados, com dúvidas, como G. iheringi.

\section{Material e Métodos}

Material examinado: NMZN 205848 e NMZN 262658 Pupa iheringi Suter,1900. BRASIL, Rio Grande do Sul, Bollaxa; Suter coll no 2176. Lectotype NMZN 205848; Paralectotype NMZN 262658, 01 ex danificado (Dados constantes na etiqueta da coleção do Museum of New Zealand - NMZN).

As medidas ( $\mathrm{mm}$ ) obtidas ao estereomicroscópio (ocular micrometrada), assim como os índices seguem Pokryszko (1996): comprimento ou altura da concha $(\mathrm{H})$, largura da concha $(\mathrm{L})$, comprimento ou altura da abertura (h), largura da abertura (l), altura da volta do corpo (vc); índices da proporção entre altura/largura da abertura da concha (H/L), altura volta do corpo/ altura da concha $(\mathrm{vc} / \mathrm{H})$. A nomenclatura para os dentes ou lâminas presentes na abertura da concha segue Pilsbry (19161918): as localizadas sobre a parede parietal e a margem columelar - lamelas; as situadas sobre a margem basal e a parede interna do lábio externo - pregas ou dobras. As fotomicrografias foram obtidas através de câmara fotográfica digital acoplada a estereomicroscópio.

\section{Redescrição do Holótipo}

Gastrocopta iheringi (Suter, 1900) (Figuras 1 a 3)

Pupa iheringi Suter, 1900: 336-337, fig. 8, 8A (Figura 5)

Gastrocopta iheringi - Pilsbry, 1916-1918: 101102; pl. 17 fig. 16; Morretes, 1949: 130; Salgado \& Coelho, 2003: 153; Simone, 2006: 116, fig. 346.

Holótipo (NMZN 205848 - Fig.1-3). Concha dextrógira, alongada, cilíndrica, delicada, lisa, coloração amarelo-palha - evidenciando apenas as linhas finas de crescimento; ápice cônico; com 5 e 1/4 de voltas arredondadas; protoconcha lisa com cerca de 1e $1 / 2$ voltas; sutura pouco profunda, porém bem marcada; volta do corpo danificada em sua face dorsal na altura da abertura (Figuras 1 e 3), corresponde à metade da altura total da concha; abertura de forma pentagonal arredondada junto à base; peristômio expandido junto a margem columelar, base e lábio externo, ornado por um conjunto de cinco dentes: lamelas parietal e angular (= parieto-angular) fusionadas formando uma plataforma 
FIGURAS 1-4 - 1-3, Gastrocopta iheringi (Suter, 1900), Holótipo (NMZN.205848): 1.vista frontal; 2. vista lateral; 3. detalhe da abertura; 4, fragmento de concha (NMZN.262658) designada, erroneamente, como paralectótipo de Gastrocopta iheringi. Barras: $1 \mathrm{~mm}$.

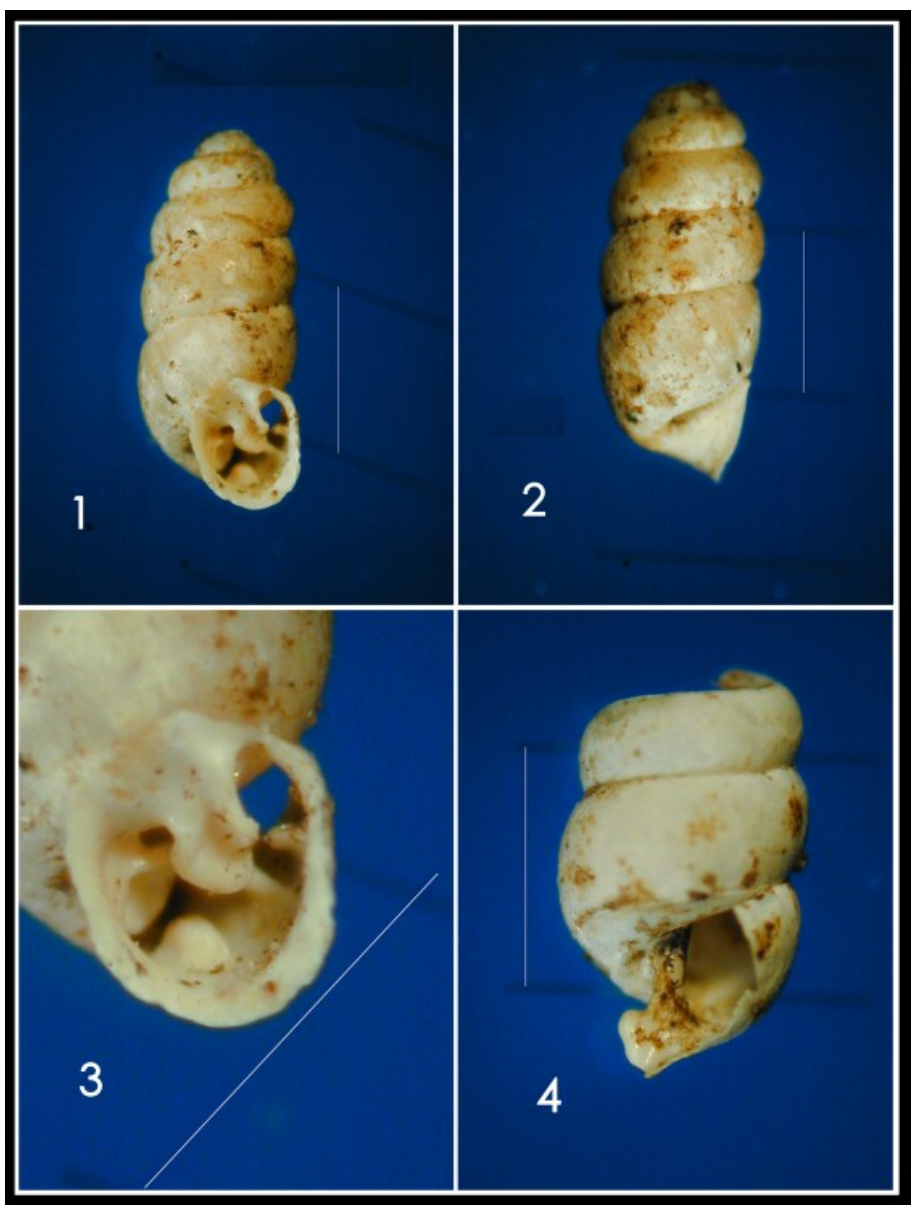

bastante proeminente que se expande em direção às dobras palatais - sobrepondo-se à dobra palatal inferior e parcialmente à lamela columelar; lamela columelar ocupa praticamente toda a margem columelar, aspecto de uma lâmina arredondada com uma concavidade; dobra basal proeminente em formato de meia lua; dobras palatais inferior e superior paralelas, sendo a dobra palatal inferior maior, projetando-se para dentro da abertura; dentes ocupam praticamente todo o perímetro interior da abertura (Figura 3); umbílico pequeno e arredondado (Figura 2). Dimensões: $\mathrm{H}=2,48 \mathrm{~mm}$; $\mathrm{L}=1,04 \mathrm{~mm} ; \mathrm{vc}=1,3 \mathrm{~mm} ; \mathrm{h}=0,60 \mathrm{~mm} ; \mathrm{l}=0,72 \mathrm{~mm}$ e as seguintes relações: $\mathrm{H} / \mathrm{L}=2,38 ; \mathrm{vc} / \mathrm{H}=0,52 ; 1 / \mathrm{vc}=0,55$.

Localidade-tipo: Bolaxa (grafia atual de Bollaxa), município de Rio Grande, Rio Grande do Sul,
Brasil [por designação original], $32^{\circ} 10^{\prime} 33,16^{\prime \prime} \mathrm{S}$ e $52^{\circ} 12^{\prime} 23,83^{\prime \prime} \mathrm{W}$.

FIGURA 5: Pupa iheringi Suter (1900, fig. 8, 8A)

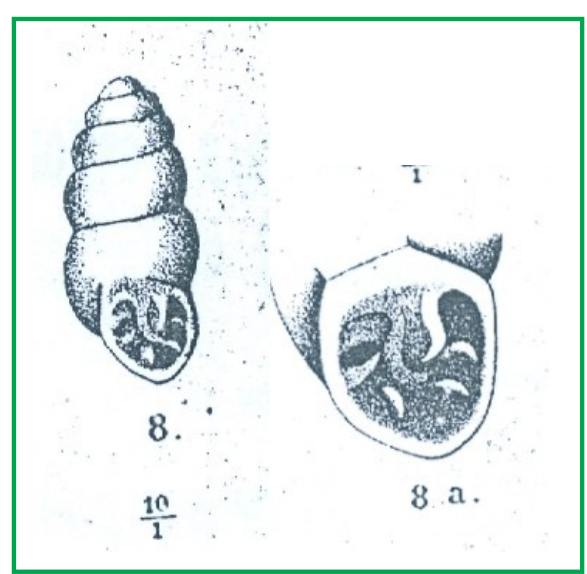




\section{Discussão}

Cabe destacar que a pequena discrepância entre as medidas de altura e a largura (=diâmetro) em relação às de Suter (1900), isto é "Diam.1., alt.2,5mm" deve-se, certamente, à precisão do equipamento utilizado pelo autor (não informado) em relação ao presente trabalho. Quanto à cor da concha, Suter (1900) caracteriza como cinzenta, tal qualidade muitas vezes reflete uma questão subjetiva.

Suter (1900) ao descrever Pupa iheringi trata a descrição, incluindo as medidas e a ilustração, com base em uma única concha, ficando isto evidente quando afirma "Typo na minha collecção. tirado em estado subfossil de depósito moderno" (grifo nosso). Em nenhum momento, Suter (1900) deixa transparecer que tenha em mãos outros exemplares deste mesmo táxon, conforme previsto pelos artigos 73.1.1 e 73.1.2 do International Commission on Zoological Nomenclature (1999), portanto deve ser tratado como holótipo por designação original.

O exame dos síntipos (2) NMZN 205848, assim designados por Marshall (1996), revelou que os mesmos foram subdivididos em dois lotes: lectótipo (M.205848) - assim designado na etiqueta que acompanha o lote , e de um paralectótipo (M.262658) - assim designado na etiqueta que acompanha o lote. Cabe ressaltar que em ambas as etiquetas - lectótipo e paralectótipo - há referência que procedem da coleção de Suter ("Suter coll' n²176"). Entretanto, Suter (1900), em sua descrição original, apenas informa "Typo na minha collecção.", não mencionando um número de coleção, a partir de tal registro se subentende tratar-se de um único exemplar.

A etiqueta do NMZN que acompanha o lote (não a original do autor) e designa o fragmento de concha como paralectótipo (NMZN 262658, concha danificada - Figura 4), não reflete a descrição de Suter (1900) que não faz menção a nenhuma outra concha e nem de um fragmento de concha que poderia ser caracterizado como de G. iheringi. Suter (1900), ao longo da descrição, original utiliza sempre o singular, inclusive ao mencionar "Typo na minha coleção", ficando tal descrição caracterizada como baseada em um único exemplar. Talvez o fragmento (Figura 4), até possa ser daquela espécie, apesar da própria abertura estar fragmentada não evidenciando a presença de dentes e lamelas. Constata-se que o autor não considerou tal fragmento em sua descrição, portanto não se justifica a designação do mesmo ou de outro qualquer exemplar como constituindo lotes da série tipo, em acordo com o artigo 72.4.1.1. do International Commission on Zoological Nomenclature (1999). O fragmento pode ter sido enviado por Ihering, juntamente como outros tantos exemplares de moluscos, conforme se pode depreender no artigo de Suter (1900), que descreve e identifica muitos outros táxons de gastrópodes terrestres enviados na mesma ocasião por H. von Ihering.

Pilsbry (1916-1918) caracteriza e ilustra (através de desenho) um exemplar depositado na Academy Natural Sciences of Philadelphia (ANSP 22940), por ele designado incorretamente como parátipo de $G$. iheringi, uma vez que tal designação somente pode ser feita quando da descrição do táxon, mesmo que as características e ilustração sejam similares ao exemplartipo (NMZN 205848). Em correspondência com o Dr. Paul Callon, responsável pelo Departamento de Malacologia da ANSP, foram solicitadas informações sobre o parátipo depositado na coleção do ANSP, conforme registrado por Pilsbry (1916-1918), e se tinha conhecimento sobre material-tipo (síntipos) depositado no NMZN. A resposta do Dr. Callon foi "I have spoken with Dr Marshall in Wellington. Apparently their specimens of Gastrocopta iheringi are catalogued as 'potential lectotype' and 'potential paralectotype', and it seems that no lectotype selection has yet taken place for this species. I am currently looking for our material and will contact you when i have it to hand." A pesquisa na coleção de Mollusca do ANSP via internet (http://clade. ansp.org/malacology/collections/search.php), mostra as seguintes informações com relação ao lote ANSP 22940, mencionado como sendo parátipo por Pilsbry (1916-1918): ANSP 22940 Bifidaria iheringi Suter; Counts: dry: 4, alc:; Original number: Locality: Rio Grande do Sul, Brazil; Depth: Elevation: Station number: Collector(s):; Date Collected:; Collection:; Date Cataloged.

Em consulta ao Dr. Luiz Ricardo L. Simone, curador da coleção de Mollusca do MUZSP, sobre a existência de tipo de G. iheringi na respectiva coleção, obtivemos a 
seguinte informação: "Não temos tipos explícitos, mas sim prováveis. Estou enviando a foto que vai sair no catálogo que estou organizando. Os dados são: "Probable Syntypes - MZSP 32872 (A) + MZSP 7519 (B); 2.5mm". De fato Simone (2006) publicou tais dados.

Frente ao exposto e considerando o artigo 73.1.1. do International Commission on Zoological Nomenclature (1999) entendemos que o único material-tipo de $G$. iheringi, a ser considerado como tal, corresponde ao exemplar NMZN 205848 e, portanto, deve ter o status de holótipo, assim fixado na designação original (Typo). Considerando, ainda, o artigo 72.4.1.1 da Commission on Zoological Nomenclature (1999), não há evidências de que o lote NMZN 262658, isto é o fragmento de uma concha de gastrópode proveniente da coleção de Suter, tenha sido considerado pelo mesmo quando da descrição de G. iheringi. Não há, portanto, nenhum outro tipo primário a ser considerado, inclusive aqueles citados por Pilsbry (1916-1918) como parátipo (ANSP 22940) e por Simone (2006) com o prováveis síntipos (MZSP 32872 (A) + MZSP 7519 (B)).

Considerando os dados constantes na etiqueta NMZN 205848, a designação original de Suter (1900) ao descrever $G$. iheringi "Cidade de Rio Grande do Sul (Bollaxa)" e agora acrescidas as coordenadas, a localidade-tipo passa a ter a seguinte configuração: Bolaxa (grafia atual de Bollaxa), município de Rio Grande, Rio Grande do Sul, Brasil [por designação original], 32¹0'33,16”'S e 52 ${ }^{\circ} 12^{\prime} 23,83$ 'W. A localidade Bolaxa consta como inserida na zona do Estuário da Lagoa dos Patos, município de Rio Grande, Rio Grande do Sul, sendo classificada como zona urbana pelo plano diretor municipal. Segundo o Núcleo de Educação e Monitoramento Ambiental - NEMA, o Sistema Arroio - Lagoa do Bolaxa apresenta tipos de vegetação bastante diversificados destacando-se as Dunas Fósseis ou Paleodunas. Tal Sistema representa a última área natural preservada dentro da zona urbana do município (CARVALHO E SILVA, 1998).

\section{Agradecimentos}

Ao Dr. B. A. Marshall pelo empréstimo dos lotes de Gastrocopta iheringi (NMZN 205848 e NMZN 262658); ao Dr. Paul Callon, pelas informações relativas ao material de G. iheringi citado por Pilsbry (1916-1918); ao Dr. Luiz R. Simone pelas informações relativas a lotes de G. iheringi da coleção do Museu de Zoologia de USP; ao Dr. José Felipe Amato pelas micrografias digitais; ao Marcelo Bordignon pelo ajuste técnico das fotos digitais.

\section{Referências}

CARVALHO, R. V.; SILVA, K. G. Caracterização ambiental do sistema Arroio-Lagoa Bolaxa - uma futura área de preservação ambiental. In: SEMANA NACIONAL DE OCEANOGRAFIA, XI, 1998, Rio Grande. Anais... Rio Grande: FURG: Rio Grande, 1998. p. 46-48.

INTERNATIONAL COMMISSION ON ZOOLOGICAL NOMENCLATURE - ICZN. International Code of Zoological Nomenclature. 4 ed. London: International Trust for Zoological Nomenclature, 1999. xxix +306 p. Disponível em <http://www. nhm.ac.uk/hosted-sites/iczn/code/>.

MARSHALL, B. A. Molluscan name-bearing types in the Museum of New Zealand Te Papa Tongarewa. Tuhinga, Wellington, n. 9, p. $1-85,1996$.

MIGUEL, S.; PARENT, H. Moluscos gasteropodos de la província de Santa fé, Argentina. Malacological Review, Michigan, v. 29, p. 107-112, 1996.

MORRETES, F. L. Ensaio de catálogo dos moluscos do Brazil. Archivos do Museu Paranaense, Curitiba, v. 7, n. 1, p. 3-216, 1949.

PILSBRY, H. A. Pupillidae (Gastrocoptinae). Manual of Concology., Philadelphia, ser II, v. 24, i-xii+380 p., pl.1-24, 19161918.

POKRYSZKO, B. M. The Vertiginidae of Poland (Gastropoda: Pulmonata: Pupilloidea): Systematics, distribution and origin. Invertebrate Taxonomy, Collingwood, v. 10, p. 1085-1150, 1996. SUTER, H. Observações sobre alguns caracóes terrestres do Brazil. Revista do Museu Paulista, São Paulo, v. 4, p. 329-337, 1900.

SAlGADO, N. C.; COELHO, A. C. S. Moluscos terrestres do Brasil (Gastrópodes operculados ou não, exclusive Veronicellidae, Milacidae e Limacidae). Revista de Biología Tropical, San José, v. 51, supl. 3, p. 149-189, 2003.

SIMONE, L. R. R. Land and freshwater mollusks of Brazil. São Paulo: EGB/FAPESP, 2006. 390 p.

THOMPSON, F. G.; LOPEZ, A. A new land snail of the genus Gastrocopta from Nicaragua (Pulmonata: Vertiginidae), and its relationship to species from northeastern South America. American Malacological Bulletin, Washington, v. 13, n. 1/2, p. 47-53, 1996. 